\title{
Casual Effect of Serum Lipid Biomarkers On Early Age-Related Macular Degeneration Using Mendelian Randomization
}

\section{Fen-Fen Li}

Eye Hospital of Wenzhou Medical University

\section{Yuqin Wang}

Eye Hospital of Wenzhou Medical University

\section{Qi Chen}

Eye Hospital of Wenzhou Medical University

\section{Lue Xiang}

Eye Hospital of Wenzhou Medical University

\section{Feng-Qin Rao}

Wenzhou Medical University

\section{Li-Jun Shen}

Wenzhou Medical University Eye Hospital

\section{Qin-Xiang Zheng}

Wenzhou Medical University Eye Hospital

\section{Quanyong Yi}

The affiliated ningbo eye hospital of Wenzhou Medical university

Xiu Feng Huang ( $\nabla$ hxfwzmc@163.com )

Wenzhou Medical University Second Affiliated Hospital https://orcid.org/0000-0002-7852-6358

\section{Research}

Keywords: serum lipid biomarkers, early age-related macular degeneration, Mendelian randomization

Posted Date: November 10th, 2021

DOI: https://doi.org/10.21203/rs.3.rs-1018388/v1

License: (c) (1) This work is licensed under a Creative Commons Attribution 4.0 International License. Read Full License 


\section{Abstract \\ Background}

Age-related macular degeneration (AMD) is one of the major causes of vision loss. Early AMD needs to be taken seriously, whereas lipid biomarkers' casual effects on early AMD remain unclear.

\section{Methods}

In this study, a two-sample Mendelian randomization (MR) analysis was performed to systematically assess the causal relationships between seven serum lipid biomarkers, consisting of apolipoprotein $A$ $(A p o A)$, apolipoprotein $B(A p o B)$, total cholesterol $(\mathrm{CHOL})$, high-density lipoprotein cholesterol (HDL-C), direct low-density lipoprotein cholesterol (LDL-C), lipoprotein A [Lp(a)], and triglycerides (TG), and the risk of early AMD. Totally, 14,034 cases and 91,214 controls of European ancestry were included in the analysis (Number of SNPs $=11,304,110)$.

\section{Results}

MR estimates showed that a higher HDL-C level was strongly associated with increased risk of early AMD $\left(\mathrm{OR}=1.25,95 \% \mathrm{Cl}: 1.15-1.35, P=2.61 \times 10^{-8}\right)$. In addition, the level of ApoA was also positively associated with the risk of early AMD $\left(\mathrm{OR}=2.04,95 \% \mathrm{Cl}: 1.50-2.77, P=6.27 \times 10^{-6}\right)$. Conversely, higher LDL-C levels significantly decreased the risk of early AMD (OR $\left.=0.90,95 \% \mathrm{Cl}: 0.85-0.96, P=2.03 \times 10^{-3}\right)$. In addition to LDL-C, higher levels of ApoB and TG were found to be positively associated with early AMD risk. Sensitivity analyses further supported these associations. Moreover, multivariable MR analyses, adjusting for the effects of correlated lipid biomarkers yielded similar results.

\section{Conclusion}

This study addresses the question of causality relationships that elevated circulating HDL-C/ApoA levels and increased risk of early AMD, whereas LDL-C, ApoB, and TG specifically reduce the risk of early AMD. These findings contribute to our better understanding of the role of lipid metabolism in drusen formation, particularly in early AMD development.

\section{Introduction}

Age-related macular degeneration (AMD) is one of the major causes of irreversible visual impairment and central vision loss in the elderly[1]. With the aging population, AMD globally is expected to climb to around 288 million by 2040[2], which poses a heavy public health burden[3]. The cause of AMD is complex, involving environmental and genetic factors that influence susceptibility to its development[1]. 
The characteristic lesions of AMD are drusen, which are formed by deposits of extracellular debris between the retinal pigment epithelium and Bruch's membrane[4,5]. Currently, the progression of AMD is classified as early, intermediate and late stage, according to the severity of fundus lesions such as drusen size and pigmentary abnormalities[6]. The pathological hallmark of early AMD is the presence of medium-size drusen ( $\geq 63 \mu \mathrm{m}$ and $<125 \mu \mathrm{m}$ ) without pigmentary abnormalities[6], which is often asymptomatic and easily ignored in the clinic. Intermediate AMD has drusen of diameter $\geq 125 \mu \mathrm{m}$ or pigmentary abnormalities[6]. Progressing to the late AMD is characterized by severe central vision loss either caused by neovascular AMD (nAMD) or geographic atrophy (GA). However, there are no effective medications for the GA subtype, the most subtypes of late AMD, and the anti-vascular endothelial growth factor (anti-VEGF) drugs for nAMD are also not curative[7]. Therefore, early screening and prompt treatment are essential to increase the likelihood of retaining functional vision.

The mechanism of drusen's initiation and formation is not yet well understood. Preclinical studies have shown that related components of drusen, including lipids and Osteopontin[8, 9]. A growing number of epidemiological studies have reported the potential role of lipids in the development of AMD[10-12]; however, the associations were inconsistent[13]. The bias in conflicting results may come from the effects of diet and medications, which are difficult to control for in patient populations[14]. In recent years, mendelian randomization (MR) has been an emerging research method to investigate the putative causal relationships between risk factors and disease using genetic variants as natural experiments[15]. Compared to traditional observational studies, MR is less likely to influence confounding factors or reverse causation[15, 16]. Existing MR studies have reported a causal relationship between increased high-density lipoprotein cholesterol (HDL-C) levels and intermediate/advanced AMD risk[17-19]. However, the relationship between serum lipid biomarkers and early AMD remains unclear.

There is a paucity of up-to-date and comprehensive information available exclusively for early AMD. Crucially, a recent meta-GWAS on early AMD included 11 sources with GWAS data and fundus photo for early AMD phenotyping, which was the largest study for early AMD study to date[20]. Hence, in this study, we systematically investigate the association between seven major serum lipid biomarkers [ApoA, ApoB, $\mathrm{CHOL}, \mathrm{HDL}-\mathrm{C}, \mathrm{LDL}-\mathrm{C}, \mathrm{Lp}(\mathrm{a})$, and TG] and the risk of early AMD by using MR analysis. Elucidating these relationships with early AMD would help us glean a better understand the role of lipids and provide lipidmodifying therapeutic targets for AMD at the early stage.

\section{Results}

\section{Associations of serum lipid biomarkers with early AMD}

Of the seven lipid-related traits, univariable MR analysis using the IVW method found five biomarkers were significantly associated with early AMD, including HDL-C, LDL-C, TG, ApoA, ApoB (Table 2). MR estimates showed that higher HDL-C levels were strongly associated with increased risk of early AMD (OR $\left.=1.25,95 \% \mathrm{Cl}: 1.15-1.35, P=2.61 \times 10^{-8}\right)$ (Figure 1A, Figure 2). Consistent results were found in the MR analysis using other three MR methods, including weighted median $\left(\mathrm{OR}=1.42, \mathrm{P}=4.51 \times 10^{-9}\right)$, weighted 
mode $\left(\mathrm{OR}=1.44, P=2.61 \times 10^{-5}\right)$, and MR Egger $\left(\mathrm{OR}=1.24, P=4.32 \times 10^{-4}\right)$. In addition, the level of ApoA was also positively associated with the risk of early AMD (OR $=2.04,95 \% \mathrm{Cl}$ : 1.50-2.77, $P=6.27 \times$ $10^{-6}$ ) (Figure 1B, Figure 2), and the estimates were supported by other three MR methods (Table 2). These results were consist with the high correlation between $\mathrm{HDL}-\mathrm{C}$ and ApoA.

Conversely, we found that higher LDL-C level significantly decreased the risk of early AMD (OR $=0.90$,

$95 \% \mathrm{Cl}: 0.85-0.96, P=2.03 \times 10^{-3}$ ) (Figure $1 \mathrm{C}$, Figure 2). However, the estimates lose significance in other three MR methods after Bonferroni correction (Table 2). In addition to LDL-C, higher levels of ApoB and TG were found to be positively associated with early AMD risk (Figure 1D-E, Figure 2). There was strong evidence for the causal association between TG and early AMD, using IVW (OR $=0.77,95 \%$ Cl: 0.71-0.84, $\left.P=5.02 \times 10^{-10}\right)$, weighted median (OR $\left.=0.78,95 \% \mathrm{Cl}: 0.68-0.88, P=8.30 \times 10^{-5}\right)$, weighted mode $(\mathrm{OR}=$ $\left.0.82,95 \% \mathrm{Cl}: 0.73-0.92, P=7.30 \times 10^{-4}\right)$, and MR Egger (OR = 0.79, 95\% Cl: $\left.0.69-0.89, P=2.14 \times 10^{-4}\right)$. Estimates from IVW and weighted mode method showed higher ApoB level was significantly associated with the deceased risk of early AMD (IVW: OR $=0.52, P=6.60 \times 10^{-5}$; weighted mode: $\mathrm{OR}=0.56, P=1.85 \times$ $10^{-3}$ ), while the results were not significant after Bonferroni correction (Table 2).

A high level of $\mathrm{CHOL}$ was nominally associated with the decreased risk of early AMD (Figure 1F, Figure 2, Table 2). No associations were observed between Lp(a) and early AMD (Supplementary Figure 1, Table 2).

\section{Sensitivity analysis}

Using the MR-Egger intercept calculation, no evidence of directional horizontal pleiotropy was observed for all lipid-related traits (intercept was close to zero with $P$ value $>0.05$ ). Results of the MR-Egger intercept were displayed in Table 2.

MR-PRESSO outlier-corrected tests were also performed in this study. We observed that MR analysis for all of the lipid-related traits showed essentially unchanged estimates and significance after removing outlier SNPs (Table 3). These outlier SNPs were mainly located in the genes that were both associated with lipid biomarkers and AMD (e.g., CETP and $A P O E$ ), thereby removing these SNPs would affect the estimated effect sizes and study power. Leave-one-out was also applied to assess the robustness of our MR findings. Leave-one-out analysis was also conducted by examining if the estimate was driven or biased by a single SNP. Finally, no outlier was observed between all seven lipid-related traits and the risk of early AMD (Figure S1-7).

\section{Multivariable MR}

We performed Multivariable MR (MVMR)-IVW analysis to evaluate the direct effects of lipid biomarkers on early AMD by conditioning on other related traits. In the classic trio (HDL-C, LDL-C and TG), the associations of HDL-C $(\mathrm{OR}=1.18, P=0.0014)$ and TG $(\mathrm{OR}=0.86, P=0.0099)$ with the risk of early AMD showed strong evidence. However, the association between LDL-C and early AMD was nominal $(P=$ 0.063). Next, HDL-C was replaced with ApoA1 in the trio (ApoA, LDL-C, and TG). The results showed ApoA 
$(\mathrm{OR}=1.85, P=0.00034)$ and $\mathrm{TG}(\mathrm{OR}=0.83, P=0.00033)$ were significantly associated with the risk of early AMD. In the group of $A p \circ A, A p o B, T G$ and $L p(a)$, the results were similar to the univariable MR analysis (ApoA: $\mathrm{OR}=1.58, P=0.0060 ; \mathrm{ApoB}: \mathrm{OR}=0.62, P=0.0086 ; \mathrm{TG}: \mathrm{OR}=0.83, P=0.00017)$.

\section{Discussion}

In this study, we conducted comprehensive MR analyses to investigate the causal effects of seven serum lipid biomarkers on the risk of early AMD. We found that higher HDL-C and ApoA levels increased the risk of early AMD, whereas LDL-C, ApoB, and TG appeared to be associated with decreased risk of early AMD. The important value of this study is to fill the gap in the causal effects of lipid biomarkers on the risk of early AMD. More importantly, these MR findings shed light on the different roles of lipid subfractions on early $A M D$ and aid us in the understanding of lipid metabolism in the initiation and formation of drusen in the early stages, as well as the potential utility of blood lipid-modifying therapies in preventing and treating AMD.

Compared with previous MR studies, the direction of causal effect size was the same as the causal effect of lipid on intermediate and late AMD, that genetically elevated HDL-C levels increased the risk of AMD[17-19]. Han et al. found that high-density HDL-C and ApoA1 levels increased the risk of intermediate, GA, CNV, and advanced AMD subtypes[19]. In our study, the effect size of HDL-C levels on early AMD was 1.25 per $1-\mathrm{mmol} / \mathrm{l}$ increase (95\% Cl: 1.22-1.70), which appeared smaller than that on intermediate AMD (OR=1.34 per 1-mmol/l increase, 95\% Cl: 1.20-1.49) [19]. Notably, there was a robust association between HDL-C and early AMD, specifically using the inverse-variance weighted, weighted median, weighted mode, and MR Egger methods. Besides, as the major apolipoproteins in HDL-C particles, our study also supported that higher ApoA levels increase the risk of early AMD, with OR 2.04 (95\%Cl: $1.50-2.77, P=6.27 \times 10^{-6}$, Table 2 ). With the ever-increasing knowledge of HDL-C, it shows two side to health. On the one hand, HDL-C induces reverse cholesterol transport associated with plaque regression and have anti-inflammatory[21] and antioxidant properties[22, 23], which could strengthen the endothelial function and decrease the risk of atherosclerosis[24]. On the other hand, the adverse effect of a high HDL-C level on AMD may partially reflect the dysfunction of HDL. It has become apparent that under certain conditions such as cardiovascular disease[25], aging[26], or an acute phase response, HDLC could have pro-inflammatory and pro-oxidant roles that damage cholesterol efflux[27, 28].

Consequently, oxidation products, such as peroxidation lipids, gradually accumulate in the retina and Bruch's membrane, which contribute to the development of AMD. Although a pilot study by Vavvas et al. over 12 months reported that high-dose atorvastatin resulted in regression of drusen deposits in 10 of 23 patients[29], the impact of lipid-modifying therapies on AMD risk is currently unknown. Our results emphasize that the potential effects of lipid-modifying drugs on AMD and related phenotypes should be further investigated.

Consistent with previous observational studies, higher TG levels reduce the risk of early AMD $[10,12]$. In our study, raised TG levels were associated with decreased risk of early AMD, with OR $0.77(95 \% \mathrm{Cl}$ : $0.71-$ $\left.0.84, P=5.02 \times 10^{-10}\right)$; the effect size was robust on MR-PRESSO outlier-corrected tests. In previous 
observational studies, LDL-C and CHOL levels were not associated with any early AMD characteristics[12]. In MR studies, the causal association between LDL-C and advanced AMD was insignificant in both Europeans and Asians[17, 18]. However, Han X et al. found that the association of LDL-C was primarily with GA (OR=0.70, 95\% Cl: 0.59-0.83, $\left.P=3.8 \times 10^{-5}\right)$ and intermediate AMD (OR=0.77, $95 \% \mathrm{Cl}$ : 0.67-0.87, $\left.P=6.5 \times 10^{-5}\right)$, but not strong enough with nAMD[19]. Similarly, ApoB (OR=0.76, 95\%Cl: 0.69-0.85) and $\mathrm{CHOL}(\mathrm{OR}=0.81,95 \% \mathrm{Cl}: 0.70-0.94)$ were also associated with decreased risk of intermediate[19]. In our study, lipids biomarkers, LDL-C (OR=0.90, 95\%Cl: $\left.0.85-0.96, P=2.03 \times 10^{-3}\right)$ and ApoB (OR=0.52, 95\% Cl: $\left.0.38-0.72, P=6.60 \times 10^{-5}\right)$ present a protective role in the development of early AMD. However, there was no robust association of LDL-C and ApoB with early AMD in weighted median analyses, suggesting that there is no consistent relationship between LDL-C and early AMD. Besides, we find no evidence of the association between $L p(a)$ and $A M D$ risk.

Dyslipidaemia has been involved in the formation of drusen, which is characterized in the early stage of AMD. Several lines of evidence support a role for dyslipidemia in AMD pathogenesis. First, histological evidence identified that drusen contains lipid material[8, 30]. Second, animal experiments have demonstrated that impaired macrophage cholesterol efflux through HDL-mediated reverse cholesterol transport may lead to a pro-angiogenic status in AMD[31,32]. Third, genetic associations study has identified risk variants in genes involved in lipid metabolism and the transfer of lipids among lipoproteins, such as hepatic lipase $C$ (LIPC), lipoprotein lipase (LPL), cholesterol ester transferase (CETP), ABC binding cassettes $A 1$ (ABCA1) and apolipoprotein $E$ (APOE), which have all been shown to influence the course of AMD pathobiology[33-35]. Moreover, previous MR studies found the dyslipidemia is causality from intermediate to late AMD[17-19]. Additionally, this study emphasis that serum lipid levels also have a causal effect on early AMD.

This study is mainly based on early AMD and provides additional insights into the causal role of seven serum lipid biomarkers. A strength of this study is that we used large-scale datasets with standard protocols to measure lipid biomarkers; this allowed us to systematically evaluate the effects of lipids on AMD risk. Compared with traditional studies, MR findings are less likely to be affected by confounding or bias from reverse causation. At the same time, our results should be interpreted in light of the limitations of the study. First, as this study was based on European ancestry participants, the generalizability of our findings to other ethnic groups requires further investigation. Second, in the MR framework, the genetically predisposed biomarker changes were assumed to have linear and lifetime effects on AMD risk. The potentially non-linear relationships and short-term effects of these biomarkers are unclear. Third, although biochemical evidence suggests that retina-specific lipid transport is facilitated by proteins regulating systemic lipid metabolism[36], retina-specific lipid concentrations would be more relevant measurements for understanding AMD risk. Further studies are needed to investigate the effects of retinaspecific lipid metabolism on AMD risk. Besides, blood lipid levels change throughout life. Therefore, longterm follow-up studies are needed to understand the impact of dyslipidemia on AMD risk and progression over time. Finally, using multiple variants is that the analysis does not point to a single mechanism being causal for AMD risk. Nevertheless, our study has important research and clinical implications. 


\section{Conclusions}

This study addresses the causality relationships that elevated circulating HDL-C/ApoA levels and increased risk of early AMD, whereas LDL-C, ApoB, and TG particularly decreased the risk of early AMD. Our results also support further investigation into whether strategies on lipid-modifying could be used to prevent or treat early AMD.

\section{Methods}

\section{Study overview}

This study used a two-sample MR approach to estimate the causal impact of exposures on the outcome using independent GWAS statistics. A total of seven lipid biomarkers, including apolipoprotein A (ApoA), apolipoprotein $B(A p o B)$, total cholesterol (CHOL), high-density lipoprotein cholesterol (HDL-C), lowdensity lipoprotein cholesterol (LDL-C), lipoprotein A [Lp(a)], and triglycerides (TG), were used as exposures and the early AMD was the outcome. All data used in the current study is publicly available GWAS, and the relevant ethical approval can be found in the corresponding studies. Multiple testing was performed by Bonferroni correction, and the significant $P$ value was of $<0.0071(=0.05 / 7)$.

\section{Genetic instruments for serum lipid biomarkers}

GWAS summary datasets of serum lipid biomarkers are from the OpenGWAS database developed by MRC Integrative Epidemiology Unit (IEU) at the University of Bristol (https://gwas.mrcieu.ac.uk/). The information of each lipid biomarker GWAS was summarised in Table 1. To ensure the association between genetic instruments and each lipid biomarker was robust, we only selected genetic variants that were at genome-wide significance $\left(P<5 \times 10^{-8}\right)$ for MR analysis. If there were more than one available GWAS datasets for one lipid biomarker, we selected the dataset with the most valid genetic instruments.

\section{GWAS summary statistics for early AMD}

The GWAS summary data of early AMD is from a recent published genome-wide association metaanalysis which successfully identified multiple novel loci[20]. Winkler and colleagues gathered GWAS data for early AMD from 11 sources, including the International AMD Genomics Consortium (IAMDGC) and UK Biobank (UKBB).[20] Totally, 14,034 cases and 91,214 controls were included in the analysis (Number of SNPs $=11,304,110)$. All individuals are of European ancestry.

\section{Statistical analysis}

To ensure only uncorrelated variants were included for MR analysis, variants correlated with the most significant SNPs were all excluded (clumping $r^{2}$ cut-off $=0.001$, clumping window $=10,000 \mathrm{~kb}$ ).

Subsequently, all included SNPs were extracted from the GWAS dataset of early AMD, and alleles were harmonized. MR analysis was conducted by using the TwoSampleMR R package[37]. We used the 
inverse-variance weighted (IVW) method as the main analysis to assess the causal effect of each lipid biomarker on early AMD.

To assess the impact of potential horizontal pleiotropy, a series of sensitivity analysis was performed, including MR-Egger regression, Weighted median method, Weighted mode method[37]. These methods allow the existence of horizontal pleiotropy but has lower statistical power than IVW[37]. Additionally, the egger intercept was calculated to assess for horizontal pleiotropy[38]. MR pleiotropy residual sum and outlier (MR-PRESSO) test was applied to assess potential bias from outliers and evaluate the overall heterogeneity[39]. Leave-one-out analysis was also applied to assess the influence of particular SNPs[37].

To avoid collinearity, we performed Multivariable MR (MVMR)-IVW analysis by selecting groups of exposures. By using genetic instruments associated with any of the included sets of exposures, MVMR analysis can estimate the influence of each exposure conditioning on the effects of the SNPs on the other related exposures.

\section{Declarations}

\section{Ethics approval and consent to participate}

All data from open access resources. GWAS summary datasets of serum lipid biomarkers are from the OpenGWAS database developed by MRC Integrative Epidemiology Unit (IEU) at the University of Bristol (https://gwas.mrcieu.ac.uk/). Winkler and colleagues gathered GWAS data for early AMD from 11 sources, including the International AMD Genomics Consortium (IAMDGC) and UK Biobank (UKBB).

Consent for publication: Not applicable.

Conflict of interest: No conflicting relationship exists for any author.

Availability of data and materials: The dataset(s) supporting the conclusions of this article is(are) included within the article (and its additional file(s)).

\section{Funding}

This work was supported by the National Natural Science Foundation of China (31771390 from XFH), and Zhejiang Province Natural Science Foundation of China (LY21H180004 from YW).

Authors' Contributions: XFH and QY contributed to the study design. XFH, FFL, YW, and QC contributed to data collection and analysis. FFL, YW, QC, LX, FQR, HYY, LJS, QY, and XFH contributed to data interpretation. XFH and FFL wrote the manuscript.

\section{Contributor Information}

Fen-Fen Li, Email: lifenfen2@qq.com 
Yuqin Wang, Email: yqwang57@163.com

Qi Chen MD, Email: qichen1983@126.com

Lue Xiang, Email: xianglue324@foxamil.com

Feng-Qin Rao, Email: fengqin3366@163.com

Lijun Shen, Email: slj@mail.eye.ac.cn

Qin-Xiang Zheng, Email: zhengqinxiang@aliyun.com

Quanyong Yi, Email: quanyong_yi@163.com

Xiu-Feng Huang, Email: hxfwzmc@163.com

\section{References}

1. Lim, L.S., P. Mitchell, J.M. Seddon, F.G. Holz and T.Y. Wong. Age-related macular degeneration. Lancet. 2012. 379(9827): p. 1728-38.DOI: 10.1016/S0140-6736(12)60282-7.

2. Wong, W.L., X. Su, X. Li, C.M. Cheung, R. Klein, C.Y. Cheng and T.Y. Wong. Global prevalence of agerelated macular degeneration and disease burden projection for 2020 and 2040: a systematic review and meta-analysis. Lancet Glob Health. 2014. 2(2): p. e106-16.DOI: 10.1016/S2214-109X(13)701451.

3. Cruess, A.F., G. Zlateva, X. Xu, G. Soubrane, D. Pauleikhoff, A. Lotery, J. Mones, R. Buggage, C. Schaefer, T. Knight, et al. Economic burden of bilateral neovascular age-related macular degeneration: multi-country observational study. Pharmacoeconomics. 2008. 26(1): p. 57-73.DOI: 10.2165/00019053-200826010-00006.

4. de Jong, P.T. Age-related macular degeneration. N Engl J Med. 2006. 355(14): p. 1474-85.DOI: 10.1056/NEJMra062326.

5. Curcio, C.A. Soft Drusen in Age-Related Macular Degeneration: Biology and Targeting Via the Oil Spill Strategies. Invest Ophthalmol Vis Sci. 2018. 59(4): p. AMD160-AMD181.DOI: 10.1167/iovs.18-24882.

6. Ferris, F.L., 3rd, C.P. Wilkinson, A. Bird, U. Chakravarthy, E. Chew, K. Csaky, S.R. Sadda and C. Beckman Initiative for Macular Research Classification. Clinical classification of age-related macular degeneration. Ophthalmology. 2013. 120(4): p. 844-51.DOI: 10.1016/j.ophtha.2012.10.036.

7. Group, C.R., D.F. Martin, M.G. Maguire, G.S. Ying, J.E. Grunwald, S.L. Fine and G.J. Jaffe. Ranibizumab and bevacizumab for neovascular age-related macular degeneration. N Engl J Med. 2011. 364(20): p. 1897-908.DOI: 10.1056/NEJMoa1102673.

8. Wang, L., M.E. Clark, D.K. Crossman, K. Kojima, J.D. Messinger, J.A. Mobley and C.A. Curcio. Abundant lipid and protein components of drusen. PLoS One. 2010. 5(4): p. e10329.DOI: 10.1371 /journal.pone.0010329. 
9. Lekwuwa, M., M. Choudhary, E.M. Lad and G. Malek. Osteopontin accumulates in basal deposits of human eyes with age-related macular degeneration and may serve as a biomarker of aging. Mod Pathol. 2021.DOI: 10.1038/s41379-021-00887-7.

10. Wang, Y., M. Wang, X. Zhang, Q. Zhang, J. Nie, M. Zhang, X. Liu and L. Ma. The Association between the Lipids Levels in Blood and Risk of Age-Related Macular Degeneration. Nutrients. 2016. 8(10).DOI: 10.3390/nu8100663.

11. Klein, R., K.E. Lee, M.Y. Tsai, K.J. Cruickshanks, R.E. Gangnon and B.E.K. Klein. Oxidized Low-density Lipoprotein and the Incidence of Age-related Macular Degeneration. Ophthalmology. 2019. 126(5): p. 752-758.DOI: 10.1016/j.ophtha.2018.12.026.

12. Colijn, J.M., A.I. den Hollander, A. Demirkan, A. Cougnard-Gregoire, T. Verzijden, E. Kersten, M.A. Meester-Smoor, B.M.J. Merle, G. Papageorgiou, S. Ahmad, et al. Increased High-Density Lipoprotein Levels Associated with Age-Related Macular Degeneration: Evidence from the EYE-RISK and European Eye Epidemiology Consortia. Ophthalmology. 2019. 126(3): p. 393-406.DOI: 10.1016/j.ophtha.2018.09.045.

13. van Leeuwen, E.M., E. Emri, B.M.J. Merle, J.M. Colijn, E. Kersten, A. Cougnard-Gregoire, S. Dammeier, M. Meester-Smoor, F.M. Pool, E.K. de Jong, et al. A new perspective on lipid research in age-related macular degeneration. Prog Retin Eye Res. 2018. 67: p. 56-86.DOI:

10.1016/j.preteyeres.2018.04.006.

14. Kelly, U.L., D. Grigsby, M.A. Cady, M. Landowski, N.P. Skiba, J. Liu, A.T. Remaley, M. Klingeborn and C. Bowes Rickman. High-density lipoproteins are a potential therapeutic target for age-related macular degeneration. J Biol Chem. 2020. 295(39): p. 13601-13616.DOI: 10.1074/jbc.RA119.012305.

15. Davies, N.M., M.V. Holmes and G. Davey Smith. Reading Mendelian randomisation studies: a guide, glossary, and checklist for clinicians. BMJ. 2018. 362: p. k601.DOI: 10.1136/bmj.k601.

16. Pingault, J.B., P.F. O'Reilly, T. Schoeler, G.B. Ploubidis, F. Rijsdijk and F. Dudbridge. Using genetic data to strengthen causal inference in observational research. Nat Rev Genet. 2018. 19(9): p. 566580.DOI: 10.1038/s41576-018-0020-3.

17. Burgess, S. and G. Davey Smith. Mendelian Randomization Implicates High-Density Lipoprotein Cholesterol-Associated Mechanisms in Etiology of Age-Related Macular Degeneration. Ophthalmology. 2017. 124(8): p. 1165-1174.DOI: 10.1016/j.ophtha.2017.03.042.

18. Fan, Q., J.C. Maranville, L. Fritsche, X. Sim, C.M.G. Cheung, L.J. Chen, M. Gorski, K. Yamashiro, J. Ahn, A. Laude, et al. HDL-cholesterol levels and risk of age-related macular degeneration: a multiethnic genetic study using Mendelian randomization. Int J Epidemiol. 2017. 46(6): p. 1891-1902.DOI: 10.1093/ije/dyx189.

19. Han, X., J.S. Ong, A.W. Hewitt, P. Gharahkhani and S. MacGregor. The effects of eight serum lipid biomarkers on age-related macular degeneration risk: a Mendelian randomization study. Int J Epidemiol. 2021. 50(1): p. 325-336.DOI: 10.1093/ije/dyaa178.

20. Winkler, T.W., F. Grassmann, C. Brandl, C. Kiel, F. Gunther, T. Strunz, L. Weidner, M.E. Zimmermann, C.A. Korb, A. Poplawski, et al. Genome-wide association meta-analysis for early age-related macular 
degeneration highlights novel loci and insights for advanced disease. BMC Med Genomics. 2020. 13(1): p. 120.DOI: 10.1186/s12920-020-00760-7.

21. Calabresi, L., M. Gomaraschi, B. Villa, L. Omoboni, C. Dmitrieff and G. Franceschini. Elevated soluble cellular adhesion molecules in subjects with low HDL-cholesterol. Arterioscler Thromb Vasc Biol. 2002. 22(4): p. 656-61.DOI: 10.1161/hq0402.105901.

22. Parthasarathy, S., J. Barnett and L.G. Fong. High-density lipoprotein inhibits the oxidative modification of low-density lipoprotein. Biochim Biophys Acta. 1990. 1044(2): p. 275-83.DOI: 10.1016/0005-2760(90)90314-n.

23. Navab, M., S.Y. Hama, G.M. Anantharamaiah, K. Hassan, G.P. Hough, A.D. Watson, S.T. Reddy, A. Sevanian, G.C. Fonarow and A.M. Fogelman. Normal high density lipoprotein inhibits three steps in the formation of mildly oxidized low density lipoprotein: steps 2 and 3. J Lipid Res. 2000. 41(9): p. 1495-508.

24. Fisher, E.A., J.E. Feig, B. Hewing, S.L. Hazen and J.D. Smith. High-density lipoprotein function, dysfunction, and reverse cholesterol transport. Arterioscler Thromb Vasc Biol. 2012. 32(12): p. 281320.DOI: 10.1161/ATVBAHA.112.300133.

25. Heinecke, J.W. The HDL proteome: a marker-and perhaps mediator-of coronary artery disease. J Lipid Res. 2009. 50 Suppl: p. S167-71.DOI: 10.1194/jlr.R800097-JLR200.

26. Holzer, M., M. Trieb, V. Konya, C. Wadsack, A. Heinemann and G. Marsche. Aging affects high-density lipoprotein composition and function. Biochim Biophys Acta. 2013. 1831(9): p. 1442-8.DOI: 10.1016/j.bbalip.2013.06.004.

27. Handa, J.T., M. Cano, L. Wang, S. Datta and T. Liu. Lipids, oxidized lipids, oxidation-specific epitopes, and Age-related Macular Degeneration. Biochim Biophys Acta Mol Cell Biol Lipids. 2017. 1862(4): p. 430-440.DOI: 10.1016/j.bbalip.2016.07.013.

28. Van Lenten, B.J., S.Y. Hama, F.C. de Beer, D.M. Stafforini, T.M. Mclntyre, S.M. Prescott, B.N. La Du, A.M. Fogelman and M. Navab. Anti-inflammatory HDL becomes pro-inflammatory during the acute phase response. Loss of protective effect of HDL against LDL oxidation in aortic wall cell cocultures. J Clin Invest. 1995. 96(6): p. 2758-67.DOI: 10.1172/JCI118345.

29. Vavvas, D.G., A.B. Daniels, Z.G. Kapsala, J.W. Goldfarb, E. Ganotakis, J.I. Loewenstein, L.H. Young, E.S. Gragoudas, D. Eliott, I.K. Kim, et al. Regression of Some High-risk Features of Age-related Macular Degeneration (AMD) in Patients Receiving Intensive Statin Treatment. EBioMedicine. 2016. 5: p. 198-203.DOI: 10.1016/j.ebiom.2016.01.033.

30. Curcio, C.A., J.B. Presley, C.L. Millican and N.E. Medeiros. Basal deposits and drusen in eyes with agerelated maculopathy: evidence for solid lipid particles. Exp Eye Res. 2005. 80(6): p. 761-75.DOI: 10.1016/j.exer.2004.09.017.

31. Sene, A., A.A. Khan, D. Cox, R.E. Nakamura, A. Santeford, B.M. Kim, R. Sidhu, M.D. Onken, J.W. Harbour, S. Hagbi-Levi, et al. Impaired cholesterol efflux in senescent macrophages promotes agerelated macular degeneration. Cell Metab. 2013. 17(4): p. 549-61.DOI: 10.1016/j.cmet.2013.03.009. 
32. Sene, A. and R.S. Apte. Eyeballing cholesterol efflux and macrophage function in disease pathogenesis. Trends Endocrinol Metab. 2014. 25(3): p. 107-14.DOI: 10.1016/j.tem.2013.10.007.

33. Chen, W., D. Stambolian, A.O. Edwards, K.E. Branham, M. Othman, J. Jakobsdottir, N. Tosakulwong, M.A. Pericak-Vance, P.A. Campochiaro, M.L. Klein, et al. Genetic variants near TIMP3 and high-density lipoprotein-associated loci influence susceptibility to age-related macular degeneration. Proc Natl Acad Sci U S A. 2010. 107(16): p. 7401-6.DOI: 10.1073/pnas.0912702107.

34. Neale, B.M., J. Fagerness, R. Reynolds, L. Sobrin, M. Parker, S. Raychaudhuri, P.L. Tan, E.C. Oh, J.E. Merriam, E. Souied, et al. Genome-wide association study of advanced age-related macular degeneration identifies a role of the hepatic lipase gene (LIPC). Proc Natl Acad Sci U S A. 2010. 107(16): p. 7395-400.DOI: 10.1073/pnas.0912019107.

35. Cheng, C.Y., K. Yamashiro, L.J. Chen, J. Ahn, L. Huang, L. Huang, C.M. Cheung, M. Miyake, P.D. Cackett, I.Y. Yeo, et al. New loci and coding variants confer risk for age-related macular degeneration in East Asians. Nat Commun. 2015. 6: p. 6063.DOI: 10.1038/ncomms7063.

36. Zheng, W., N. Mast, A. Saadane and I.A. Pikuleva. Pathways of cholesterol homeostasis in mouse retina responsive to dietary and pharmacologic treatments. J Lipid Res. 2015. 56(1): p. 81-97.DOI: 10.1194/jlr.M053439.

37. Hemani, G., J. Zheng, B. Elsworth, K.H. Wade, V. Haberland, D. Baird, C. Laurin, S. Burgess, J. Bowden, R. Langdon, et al. The MR-Base platform supports systematic causal inference across the human phenome. Elife. 2018. 7.DOI: 10.7554/eLife.34408.

38. Bowden, J., G. Davey Smith and S. Burgess. Mendelian randomization with invalid instruments: effect estimation and bias detection through Egger regression. Int J Epidemiol. 2015. 44(2): p. 51225.DOI: $10.1093 / \mathrm{ije} / \mathrm{dyv} 080$.

39. Verbanck, M., C.Y. Chen, B. Neale and R. Do. Detection of widespread horizontal pleiotropy in causal relationships inferred from Mendelian randomization between complex traits and diseases. Nat Genet. 2018. 50(5): p. 693-698.DOI: 10.1038/s41588-018-0099-7.

\section{Tables}

Table 1. Description of the GWAS statistics of lipid biomarkers. 


\begin{tabular}{|lllll|}
\hline Trait & GWAS accession number & Sample size & Number of SNPs & Population \\
\hline ApoA & ukb-d-30630_raw & NA & $13,585,234$ & European \\
\hline ApoB & ukb-d-30640_raw & NA & $13,585,958$ & European \\
\hline CHOL & ieu-a-301 & 187,365 & $2,446,982$ & Mixed \\
\hline HDL-C & ieu-b-109 & 403,943 & $12,321,875$ & European \\
\hline LDL-C & ebi-a-GCST90002412 & 431,167 & $16,293,344$ & European \\
\hline Lp(a) & ukb-d-30790_raw & NA & $13,583,854$ & European \\
\hline TG & ieu-b-111 & 441,016 & $12,321,875$ & European \\
\hline
\end{tabular}

Table 2. Casual effect of lipid biomarkers on early AMD. 


\begin{tabular}{|c|c|c|c|c|c|c|c|}
\hline Exposure & SNPs & Method & Estimate & $\begin{array}{l}\text { Standard } \\
\text { Error }\end{array}$ & P-value & OR & $95 \% \mathrm{C}$ \\
\hline & & $\begin{array}{l}\text { Inverse variance } \\
\text { weighted }\end{array}$ & 0.71 & 0.16 & $\begin{array}{l}6.27 \times \\
10^{-6}\end{array}$ & 2.04 & $\begin{array}{l}1.50- \\
2.77\end{array}$ \\
\hline & & Weighted median & 0.91 & 0.28 & $\begin{array}{l}1.35 \times \\
10^{-3}\end{array}$ & 2.48 & $\begin{array}{l}1.42- \\
4.32\end{array}$ \\
\hline \multirow[t]{5}{*}{ ApoA } & 237 & Weighted mode & 1.46 & 0.39 & $\begin{array}{l}2.07 \times \\
10^{-4}\end{array}$ & 4.33 & $\begin{array}{l}2.02- \\
9.27\end{array}$ \\
\hline & & MR Egger & 0.83 & 0.24 & $\begin{array}{l}7.15 \times \\
10^{-4}\end{array}$ & 2.28 & $\begin{array}{l}1.42- \\
3.66\end{array}$ \\
\hline & & (intercept) & $\begin{array}{l}-1.41 \times \\
10^{-3}\end{array}$ & $2.25 \times 10^{-3}$ & $\begin{array}{l}5.33 \times \\
10^{-1}\end{array}$ & & \\
\hline & & $\begin{array}{l}\text { Inverse variance } \\
\text { weighted }\end{array}$ & -0.65 & 0.16 & $\begin{array}{l}6.60 \times \\
10^{-5}\end{array}$ & 0.52 & $\begin{array}{l}0.38- \\
0.72\end{array}$ \\
\hline & & Weighted median & -0.54 & 0.23 & $\begin{array}{l}1.84 \times \\
10^{-2}\end{array}$ & 0.58 & $\begin{array}{l}0.37- \\
0.91\end{array}$ \\
\hline \multirow[t]{5}{*}{ ApoB } & 166 & Weighted mode & -0.58 & 0.18 & $\begin{array}{l}1.85 \times \\
10^{-3}\end{array}$ & 0.56 & $\begin{array}{l}0.39- \\
0.80\end{array}$ \\
\hline & & MR Egger & -0.52 & 0.22 & $\begin{array}{l}1.67 \times \\
10^{-2}\end{array}$ & 0.59 & $\begin{array}{l}0.39- \\
0.91\end{array}$ \\
\hline & & (intercept) & $\begin{array}{l}-2.06 \times \\
10^{-3}\end{array}$ & $2.28 \times 10^{-3}$ & $\begin{array}{l}3.69 \times \\
10^{-1}\end{array}$ & & \\
\hline & & $\begin{array}{l}\text { Inverse variance } \\
\text { weighted }\end{array}$ & -0.10 & 0.05 & $\begin{array}{l}4.50 \times \\
10^{-2}\end{array}$ & 0.90 & $\begin{array}{l}0.82- \\
1.00\end{array}$ \\
\hline & & Weighted median & -0.18 & 0.06 & $\begin{array}{l}5.40 \times \\
10^{-3}\end{array}$ & 0.84 & $\begin{array}{l}0.74- \\
0.95\end{array}$ \\
\hline \multirow[t]{5}{*}{ CHOL } & 86 & Weighted mode & -0.24 & 0.06 & $\begin{array}{l}6.56 \times \\
10^{-5}\end{array}$ & 0.79 & $\begin{array}{l}0.70- \\
0.88\end{array}$ \\
\hline & & MR Egger & -0.21 & 0.08 & $\begin{array}{l}1.34 \times \\
10^{-2}\end{array}$ & 0.81 & $\begin{array}{l}0.69- \\
0.95\end{array}$ \\
\hline & & (intercept) & $\begin{array}{l}7.45 \times \\
10^{-3}\end{array}$ & $4.58 \times 10^{-3}$ & $\begin{array}{l}1.08 \times \\
10^{-1}\end{array}$ & & \\
\hline & & $\begin{array}{l}\text { Inverse variance } \\
\text { weighted }\end{array}$ & 0.22 & 0.04 & $\begin{array}{l}2.61 \times \\
10^{-8}\end{array}$ & 1.25 & $\begin{array}{l}1.15- \\
1.35\end{array}$ \\
\hline & & Weighted median & 0.35 & 0.06 & $\begin{array}{l}4.51 \times \\
10^{-9}\end{array}$ & 1.42 & $\begin{array}{l}1.26- \\
1.59\end{array}$ \\
\hline
\end{tabular}




\begin{tabular}{|c|c|c|c|c|c|c|c|}
\hline \multirow[t]{5}{*}{ HDL-C } & 344 & Weighted mode & 0.36 & 0.09 & $\begin{array}{l}2.61 \times \\
10^{-5}\end{array}$ & 1.44 & $\begin{array}{l}1.22- \\
1.70\end{array}$ \\
\hline & & MR Egger & 0.22 & 0.06 & $\begin{array}{l}4.32 \times \\
10^{-4}\end{array}$ & 1.24 & $\begin{array}{l}1.10- \\
1.40\end{array}$ \\
\hline & & (intercept) & $\begin{array}{l}1.73 \times \\
10^{-4}\end{array}$ & $1.80 \times 10^{-3}$ & $\begin{array}{l}9.23 \times \\
10^{-1}\end{array}$ & & \\
\hline & & $\begin{array}{l}\text { Inverse variance } \\
\text { weighted }\end{array}$ & -0.10 & 0.03 & $\begin{array}{l}2.03 \times \\
10^{-3}\end{array}$ & 0.90 & $\begin{array}{l}0.85- \\
0.96\end{array}$ \\
\hline & & Weighted median & -0.08 & 0.05 & $\begin{array}{l}8.02 \times \\
10^{-2}\end{array}$ & 0.92 & $\begin{array}{l}0.84- \\
1.01\end{array}$ \\
\hline \multirow[t]{5}{*}{ LDL-C } & 350 & Weighted mode & -0.11 & 0.04 & $\begin{array}{l}9.34 \times \\
10^{-3}\end{array}$ & 0.90 & $\begin{array}{l}0.82- \\
0.97\end{array}$ \\
\hline & & MR Egger & -0.11 & 0.05 & $\begin{array}{l}1.68 \times \\
10^{-2}\end{array}$ & 0.90 & $\begin{array}{l}0.82- \\
0.98\end{array}$ \\
\hline & & (intercept) & $\begin{array}{l}4.04 \times \\
10^{-4}\end{array}$ & $1.66 \times 10^{-3}$ & $\begin{array}{l}8.08 \times \\
10^{-1}\end{array}$ & & \\
\hline & & $\begin{array}{l}\text { Inverse variance } \\
\text { weighted }\end{array}$ & $\begin{array}{l}-1.10 \times \\
10^{-4}\end{array}$ & $5.43 \times 10^{-4}$ & $\begin{array}{l}8.39 \times \\
10^{-1}\end{array}$ & 1 & $\begin{array}{l}0.999- \\
1.001\end{array}$ \\
\hline & & Weighted median & $\begin{array}{l}2.06 \times \\
10^{-5}\end{array}$ & $6.39 \times 10^{-4}$ & $\begin{array}{l}9.74 \times \\
10^{-1}\end{array}$ & 1 & $\begin{array}{l}0.999- \\
1.001\end{array}$ \\
\hline \multirow[t]{5}{*}{ Lp(a) } & 19 & Weighted mode & $\begin{array}{l}-3.76 \times \\
10^{-6}\end{array}$ & $5.92 \times 10^{-4}$ & $\begin{array}{l}9.95 \times \\
10^{-1}\end{array}$ & 1 & $\begin{array}{l}0.999- \\
1.001\end{array}$ \\
\hline & & MR Egger & $\begin{array}{l}7.27 \times \\
10^{-4}\end{array}$ & $7.91 \times 10^{-4}$ & $\begin{array}{l}3.71 \times \\
10^{-1}\end{array}$ & 1.001 & $\begin{array}{l}0.999- \\
1.002\end{array}$ \\
\hline & & (intercept) & $\begin{array}{l}-1.73 \times \\
10^{-2}\end{array}$ & $1.22 \times 10^{-2}$ & $\begin{array}{l}1.73 \times \\
10^{-1}\end{array}$ & & \\
\hline & & $\begin{array}{l}\text { Inverse variance } \\
\text { weighted }\end{array}$ & -0.26 & 0.04 & $\begin{array}{l}5.02 \times \\
10^{-10}\end{array}$ & 0.77 & $\begin{array}{l}0.71- \\
0.84\end{array}$ \\
\hline & & Weighted median & -0.25 & 0.06 & $\begin{array}{l}8.30 \times \\
10^{-5}\end{array}$ & 0.78 & $\begin{array}{l}0.68- \\
0.88\end{array}$ \\
\hline \multirow[t]{3}{*}{ TG } & 295 & Weighted mode & -0.20 & 0.06 & $\begin{array}{l}7.30 \times \\
10^{-4}\end{array}$ & 0.82 & $\begin{array}{l}0.73- \\
0.92\end{array}$ \\
\hline & & MR Egger & -0.24 & 0.06 & $\begin{array}{l}2.14 \times \\
10^{-4}\end{array}$ & 0.79 & $\begin{array}{l}0.69- \\
0.89\end{array}$ \\
\hline & & (intercept) & $\begin{array}{l}-8.18 \times \\
10^{-4}\end{array}$ & $1.91 \times 10^{-3}$ & $\begin{array}{l}6.69 \times \\
10^{-1}\end{array}$ & & \\
\hline
\end{tabular}


$\mathrm{AMD}=$ age-related macular degeneration, $\mathrm{Cl}=$ confidence interval, $\mathrm{MR}=$ Mendelian randomization, $\mathrm{OR}=\mathrm{odds}$ ratio.

$\mathrm{P}<0.0071$ considered as significant at genome-wide association level and with bolded numbers.

Table 3. MR-PRESSO estimates of the associations between seven serum lipid biomarkers and early agerelated macular degeneration.

\begin{tabular}{|lllll|}
\hline Exposure & MR-PRESSO method & Causal Estimate & P-value & Distortion P value \\
\hline ApoA & Raw & 0.71 & $9.91 \times 10^{-6}$ & 0.64 \\
\hline ApoA & Outlier-corrected & 0.65 & $2.33 \times 10^{-4}$ & 0.64 \\
\hline ApoB & Raw & -0.65 & $1.00 \times 10^{-4}$ & 0.45 \\
\hline ApoB & Outlier-corrected & -0.57 & $2.97 \times 10^{-4}$ & 0.45 \\
\hline CHOL & Raw & -0.10 & $4.82 \times 10^{-2}$ & 0.08 \\
\hline CHOL & Outlier-corrected & -0.06 & $2.02 \times 10^{-1}$ & 0.08 \\
\hline HDL-C & Raw & 0.22 & $5.28 \times 10^{-8}$ & 0.26 \\
\hline HDL-C & Outlier-corrected & 0.28 & $1.67 \times 10^{-12}$ & 0.26 \\
\hline LDL-C & Raw & -0.10 & $2.20 \times 10^{-3}$ & 0.79 \\
\hline LDL-C & Outlier-corrected & -0.09 & $2.96 \times 10^{-3}$ & 0.79 \\
\hline Lp(a) & Raw & 0.0004 & $1.92 \times 10^{-1}$ & NA \\
\hline Lp(a) & Outlier-corrected & NA & NA & NA \\
\hline TG & Raw & -0.26 & $1.72 \times 10^{-9}$ & 0.94 \\
\hline TG & Outlier-corrected & -0.26 & $2.24 \times 10^{-10}$ & 0.94 \\
\hline
\end{tabular}

$\mathrm{P}<0.0071$ considered as significant at genome-wide association level and with bolded numbers.

\section{Figures}



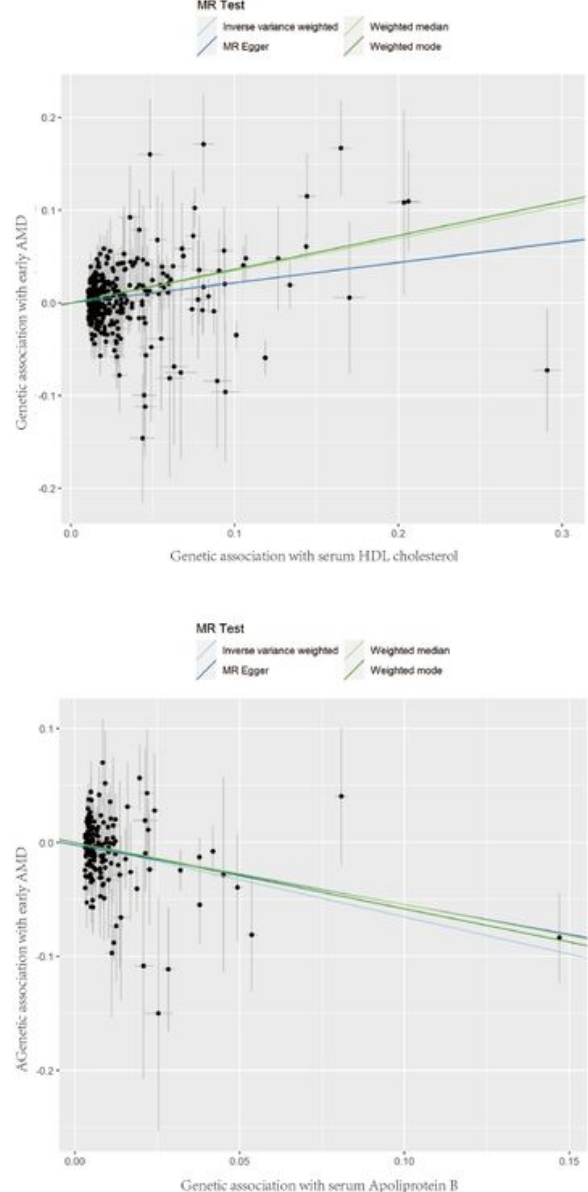

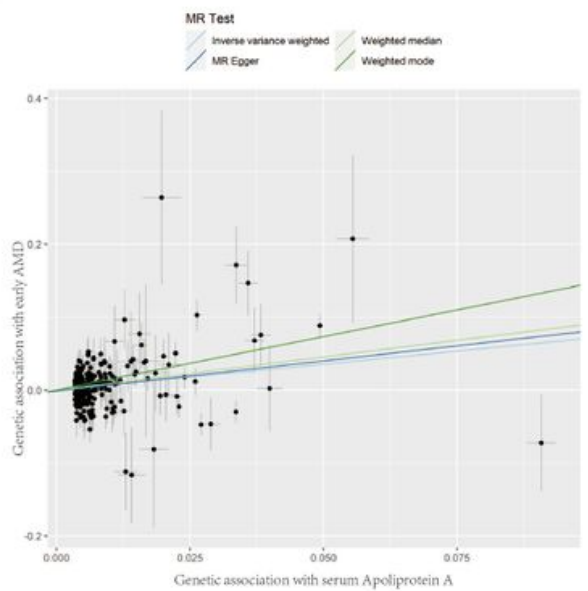

E

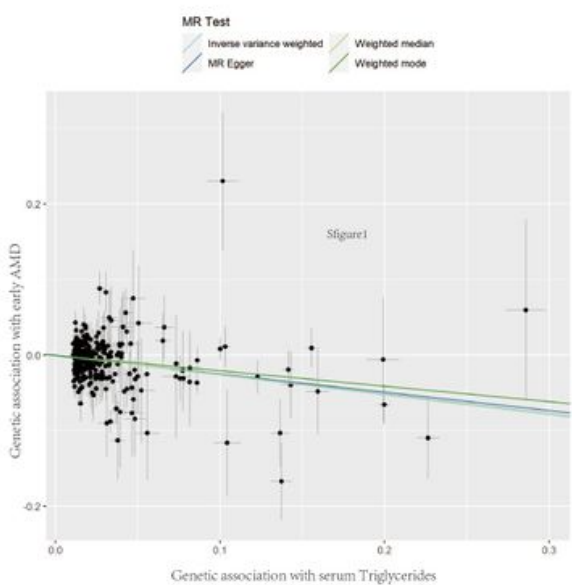

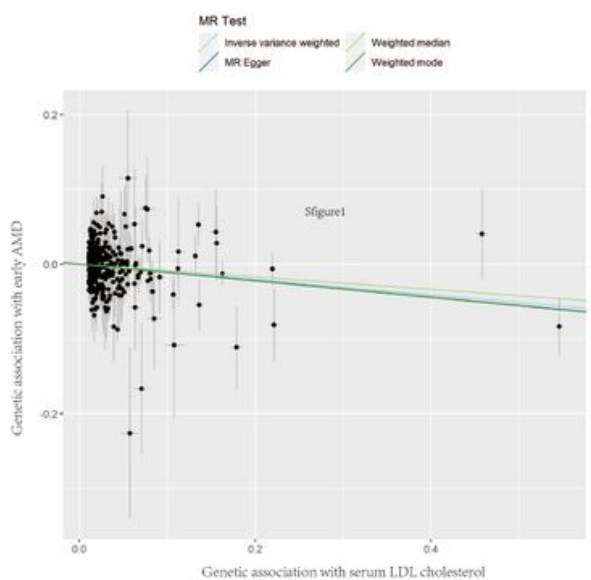

F
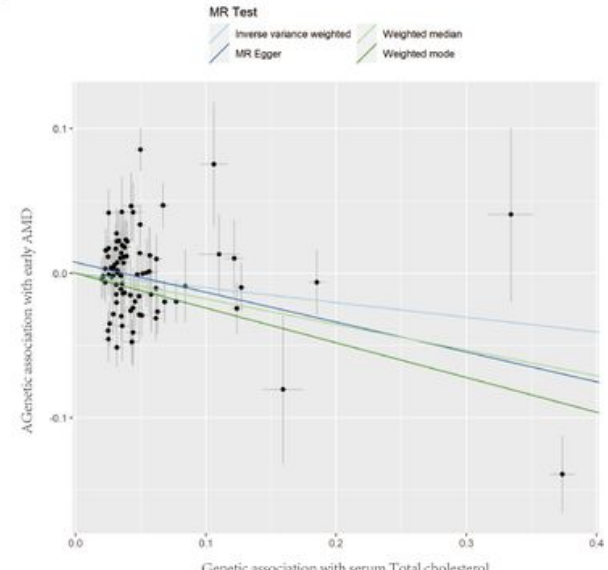

\section{Figure 1}

The causal relationships between serum lipid biomarkers and the risk of early AMD. The causal relationships between serum HDL-C (A), ApoA (B), LDL-C (C), ApoB (D), TG (E), and CHOL (F) and the risk of early AMD, respectively. The $x$-axis shows the estimated for serum lipid levels, and the $y$-axis shows the estimates (log odd ratios) of the effects on early AMD. the MR-IVW, MR-Egger, simple median and weighted median method lines are plotted with different colours. 


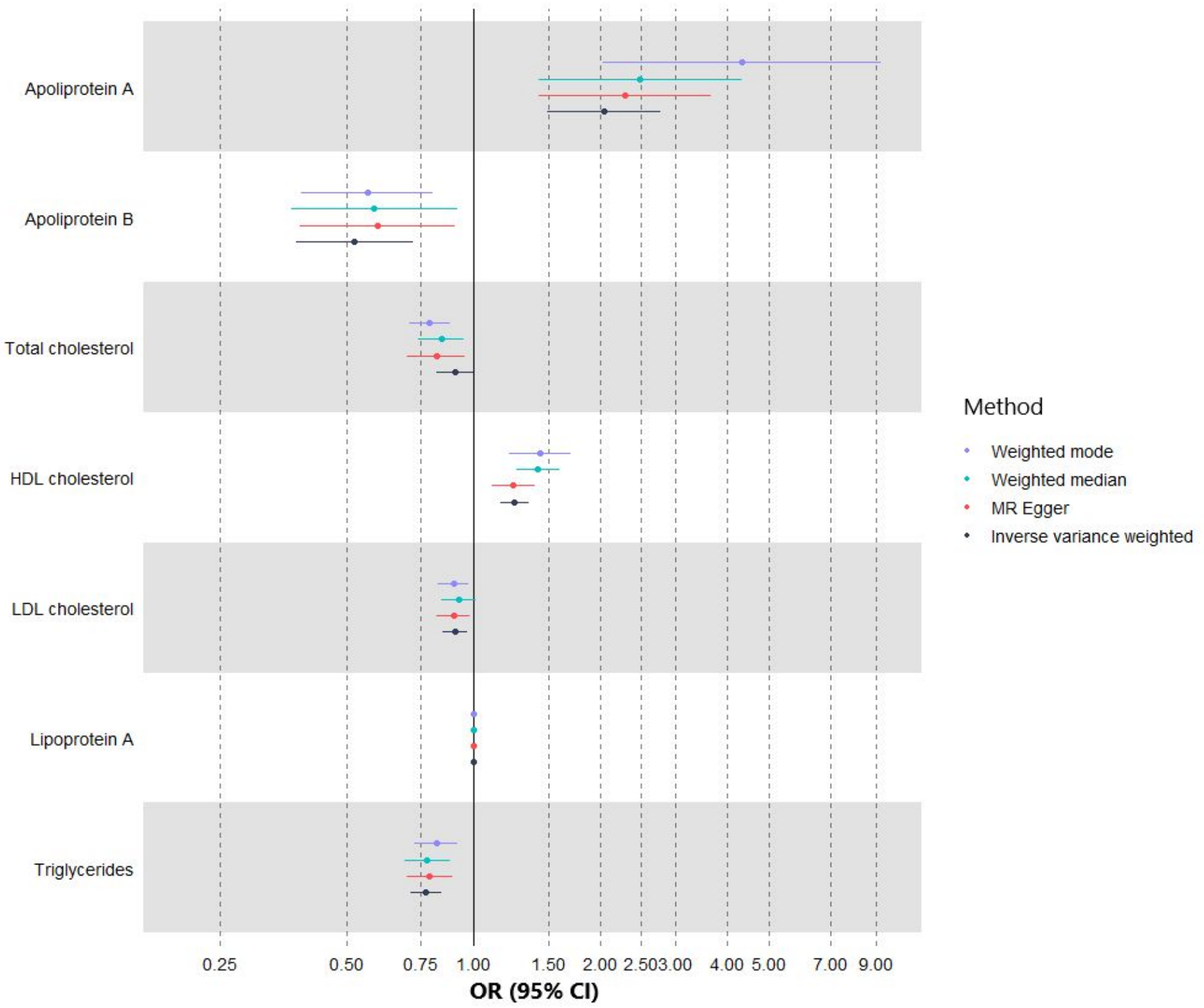

\section{Figure 2}

Univariable MR estimates of the associations between seven serum lipid biomarkers and early AMD. The vertical line is the reference at $\mathrm{OR}=1$. Different MR methods are displayed with different colours.

\section{Supplementary Files}

This is a list of supplementary files associated with this preprint. Click to download.

- supplementarylff20210919.pdf 